Bull. Korean Math. Soc. 48 (2011), No. 5, pp. 1047-1061

http://dx.doi.org/10.4134/BKMS.2011.48.5.1047

\title{
EXISTENCE AND UNIQUENESS OF POSITIVE SOLUTIONS FOR SECOND-ORDER STURM-LIOUVILLE AND MULTI-POINT PROBLEMS ON TIME SCALES
}

\author{
Yanbin Sang, Zhongli Wei, and Wei Dong
}

\begin{abstract}
In this paper, a class of second-order boundary value problems with Sturm-Liouville boundary conditions or multi-point conditions is considered. Some existence and uniqueness theorems of positive solutions of the problem are obtained by using monotone iterative technique, the iterative sequences yielding approximate solutions are also given. The results are illustrated with an example.
\end{abstract}

\section{Introduction}

In this paper, we are interested in the existence, uniqueness and iteration of positive solutions for the following second-order boundary value problem with Sturm-Liouville boundary conditions or multi-point conditions on time scales

$$
\begin{gathered}
\left(p y^{\Delta}\right)^{\nabla}(t)+h(t) f(y(t))=0, \quad t \in(a, b]_{\mathbb{T}}, \\
\alpha y(a)-\beta\left(p y^{\Delta}\right)(a)=0, \quad \gamma y^{\sigma}(b)+\delta\left(p y^{\Delta}\right)(b)=0, \quad \text { or }
\end{gathered}
$$

$$
\alpha y(a)-\beta\left(p y^{\Delta}\right)(a)=\sum_{i=1}^{n} \phi_{i}\left(p y^{\Delta}\right)\left(t_{i}\right), \quad \gamma y^{\sigma}(b)+\delta\left(p y^{\Delta}\right)(b)=\sum_{i=1}^{n} \psi_{i}\left(p y^{\Delta}\right)\left(t_{i}\right),
$$

where

$$
p:[a, \sigma(b)]_{\mathbb{T}} \longrightarrow(0,+\infty), \quad p \in C[a, \sigma(b)]_{\mathbb{T}},
$$

$$
\beta, \delta \in(0,+\infty), \quad \alpha, \gamma \in[0,+\infty), \quad \beta \gamma+\alpha \delta+\alpha \gamma \int_{a}^{\sigma(b)} \frac{\Delta \tau}{p(\tau)}>0,
$$

Received April 1, 2010; Revised July 19, 2010.

2010 Mathematics Subject Classification. 34B15, 39A10.

Key words and phrases. time scales, positive solutions, uniqueness, fixed point, monotone iterative technique.

The authors were supported financially by the National Natural Science Foundation of China (10971046), the Natural Science Foundation of Shandong Province (ZR2009AM004), the Youth Science Foundation of Shanxi Province (2009021001-2), the National Sciences Foundation of China (10901145), and the Top Young Academic Leaders of Higher Learning Institutions of Shanxi. 
the points $t_{i} \in(a, b)_{\mathbb{T}}$ for $i \in\{1,2, \ldots, n\}$ with $t_{1}<t_{2}<\cdots<t_{n}$, the scalar constants $\phi_{i}$ and $\psi_{i}$ are in $[0,+\infty)$ for $i \in\{1, \ldots, n\}$. Some preliminary definitions and theorems on time scales can be seen in $[1,9,10,11]$ which are excellent references for the calculus of time scales.

The study of dynamic equations on time scales goes back to its founder Hilger [14], and is a new area of still fairly theoretical exploration in mathematics. In particular, the theory is widely applied to biology, heat transfer, epidemic models and stock market, for details, see [8, 14, 15, 23, 26] and references therein. In recent years, there was much attention focused on the existence of positive solutions of second-order boundary value problems on time scales, such investigations can provide accurate information of phenomena that manifest themselves partly in continuous time and partly in discrete time. We refer the reader to $[1-7,12,13,16,17,19,20,22,24]$ for some recent results. But very little work has been done on the uniqueness and iteration of positive solutions of dynamic equations on time scales. We would like to mention some results of Anderson and Wong [6], Jankowski [16] and Wang, Wu and Wu [24], which motivated us to consider the BVP (1.1), (1.2) and (1.3).

In [6], Anderson and Wong studied the second-order time scale semipositone boundary value problem

$$
\left(p y^{\Delta}\right)^{\nabla}(t)+\lambda f(t, u(t))=0, \quad t \in(a, b]_{\mathbb{T}},
$$

with Sturm-Liouville boundary conditions (1.2) or multi-point conditions (1.3).

On the other hand, more and more authors have proved the existence results for dynamic equations on time scales by using the method of lower and upper solutions. In [16], Jankowski investigated second order dynamic equations with deviating arguments on time scales of the form

$$
\begin{gathered}
-x^{\Delta \Delta}(t)=f(t, x(t), x(\alpha(t))) \equiv(F x)(t), t \in[0, T]_{\mathbb{T}}, \\
x(0)=k_{1} \in \mathbb{R}, \quad x(T)=k_{2} \in \mathbb{R} .
\end{gathered}
$$

They formulated sufficient conditions, under which such problems had a minimal and a maximal solution in a corresponding region bounded by upper-lower solutions.

In [24], Wang, Wu and Wu considered a method of generalized quasilinearization, with even-order $k(k \geq 2)$ convergence, for the BVP

$$
\begin{gathered}
-\left(p(t) x^{\Delta}\right)^{\nabla}+q(t) x^{\sigma}=f\left(t, x^{\sigma}\right)+g\left(t, x^{\sigma}\right), \quad t \in[a, b]_{\mathbb{T}}, \\
\tau_{1} x(\rho(a))-\tau_{2} x^{\Delta}(\rho(a))=0, \quad x(\sigma(b))-\tau_{3} x(\eta)=0 .
\end{gathered}
$$

The main contribution in [24] relaxed the monotone conditions on $f^{(i)}(t, x)$, $g^{(i)}(t, x)(1<i<k)$ including a more general concept of upper and lower solution in mathematical biology, so that the high-order convergence of the iterations was ensured for a larger class of nonlinear functions on time scales. 
We would also like to mention the results of Sang [20] and Sun [22]. In [20], we considered the existence of positive solutions and established the corresponding iterative schemes for the following $m$-point boundary value problem on time scales

$$
\begin{gathered}
u^{\Delta \nabla}(t)+f(t, u(t))=0, \quad t \in[0,1] \subset \mathbb{T}, \\
\beta u(0)-\gamma u^{\Delta}(0)=0, u(1)=\sum_{i=1}^{m-2} \alpha_{i} u\left(\xi_{i}\right), \quad m \geq 3 .
\end{gathered}
$$

By considering the "heights" of the nonlinear term $f$ on some bounded sets and applying monotone iterative techniques on a Banach space, we did not only obtain the existence of positive solutions for the BVP (1.11) and (1.12), but also established the iterative schemes for approximating the solutions. Our method was based on the associated Green's function obtained in [13]. In essence, we combined the method of lower and upper solutions with the cone expansion and compression fixed point theorem of norm type.

Very recently, in [22], Sun investigated the existence of at least one positive solution for the following second-order three-point BVP on time scales

$$
\begin{gathered}
-x^{\Delta \Delta}(t)=f(t, x(t)), \quad t \in[a, b]_{\mathbb{T}}, \\
x(a)=0, \quad x\left(\sigma^{2}(b)\right)=\delta x(\eta),
\end{gathered}
$$

where $a, b \in \mathbb{T}$ with $a<b, \eta \in(a, b)_{\mathbb{T}}$ and $0<\delta<\frac{\sigma^{2}(b)-a}{\eta-a}$.

For the function $f$, the author imposed the following hypotheses:

$\left(A_{1}\right) f:[a, b]_{\mathbb{T}} \times \mathbb{R}^{+} \longrightarrow \mathbb{R}^{+}$is continuous;

$\left(A_{2}\right)$ for fixed $t \in[a, b]_{\mathbb{T}}, f(t, u)$ is monotone increasing on $u$;

$\left(A_{3}\right)$ there exists $q \in(0,1)$ such that

$$
f(t, r u) \geq r^{q} f(t, u) \text { for } r \in(0,1) \text { and }(t, u) \in[a, b]_{\mathbb{T}} \times \mathbb{R}^{+} .
$$

The author obtained the following result.

Theorem 1.1 ([22, Theorem 2.3]). Assume that $\left(A_{1}\right)-\left(A_{3}\right)$ are satisfied. Then the BVP (1.13) and (1.14) has at least one positive solution $\omega$. Furthermore, there exist $M \geq m>0$ such that

$$
m(t-a) \leq \omega(t) \leq M(t-a), \quad t \in\left[a, \sigma^{2}(b)\right]_{\mathbb{T}} .
$$

In this work, without demanding the existence of upper and lower solutions, we present conditions for the BVP (1.1), (1.2) and (1.3) to have a unique solution and then study the convergence of the iterative sequence. Furthermore, we study the existence and uniqueness of positive solutions of the BVP (1.13) and (1.14). Our result obtained here improves Theorem 1.1. The ideas come from Zhai [25] and Liang [18]. 
Let $\mathbb{T}$ be a time scale which has the subspace topology inherited from the standard topology on $\mathbb{R}$. For each interval $I$ of $\mathbb{R}$, we define $I_{\mathbb{T}}=I \cap \mathbb{T}$. Let the Banach space $\mathbb{B}=C[a, \sigma(b)]_{\mathbb{T}}$ be equipped with the norm

$$
\|u\|=\sup _{t \in[a, \sigma(b)]_{\mathbb{T}}}|u(t)| .
$$

Throughout this paper, we need the following assumptions:

$\left(H_{1}\right) f:[0,+\infty) \longrightarrow[0,+\infty)$ is continuous and $f(y)>0$ for $y>0$;

$\left(H_{2}\right) h:(a, b]_{\mathbb{T}} \longrightarrow[0,+\infty)$ is continuous and $\int_{a}^{b} G(s, s) h(s) \nabla s>0$, where $G(s, s)$ is given in $(2.3)$;

$\left(H_{3}\right) f:[0,+\infty) \longrightarrow[0,+\infty)$ is nonincreasing, and there exist two positivevalue functions $\varphi(\tau), \omega(\tau)$ on interval $(a, b)_{\mathbb{T}}$ such that $\varphi:(a, b)_{\mathbb{T}} \longrightarrow(0,1)$ is a surjection and $\omega(\tau)>\varphi(\tau), \forall \tau \in(a, b)_{\mathbb{T}}$ which satisfy $f\left(\frac{1}{\varphi(\tau)} y\right) \geq$ $\omega(\tau) f(y), \quad \forall \tau \in(a, b)_{\mathbb{T}}, \quad y \geq 0$.

\section{Several lemmas}

To prove the main results in this paper, we will employ several lemmas. These lemmas are based on the linear equation

$$
-\left(p y^{\Delta}\right)^{\nabla}(t)=u(t), \quad t \in(a, b]_{\mathbb{T}},
$$

with boundary conditions (1.2) and (1.3). Define the constant $d$ via

$$
d:=\beta \gamma+\alpha \delta+\alpha \gamma \int_{a}^{\sigma(b)} \frac{\Delta \tau}{p(\tau)} .
$$

Lemma 2.1 ([6, Lemma 2.1]). Assume (1.4) and (1.5). Then the nonhomogeneous boundary value problem (2.1), (1.2) has a unique solution $y$ for which the formula

$$
y(t)=\int_{a}^{b} G(t, s) u(s) \nabla s, \quad t \in[a, \sigma(b)]_{\mathbb{T}}
$$

holds, where the Green function $G(t, s)$ is given by

$$
G(t, s)=\frac{1}{d} \begin{cases}\left(\beta+\alpha \int_{a}^{s} \frac{\Delta \tau}{p(\tau)}\right)\left(\delta+\gamma \int_{t}^{\sigma(b)} \frac{\Delta \tau}{p(\tau)}\right): & a \leq s \leq t \leq \sigma(b), \\ \left(\beta+\alpha \int_{a}^{t} \frac{\Delta \tau}{p(\tau)}\right)\left(\delta+\gamma \int_{s}^{\sigma(b)} \frac{\Delta \tau}{p(\tau)}\right): & a \leq t \leq s \leq \sigma(b),\end{cases}
$$

for all $t, s \in[a, \sigma(b)]_{\mathbb{T}}$, where $d$ is given in $(2.2)$.

Lemma 2.2 ([6, Lemma 2.2]). Assume (1.4) and (1.5). Then the Green function $G(t, s)$ in $(2.3)$ satisfies

$$
g(t) G(s, s) \leq G(t, s) \leq G(s, s), \quad t, s \in[a, \sigma(b)]_{\mathbb{T}},
$$


where $g$ is given by

$$
g(t)=\min _{t \in[a, \sigma(b)]_{\mathbb{T}}}\left\{\frac{\delta+\gamma \int_{t}^{\sigma(b)} \frac{\Delta \tau}{p(\tau)}}{\delta+\gamma \int_{a}^{\sigma(b)} \frac{\Delta \tau}{p(\tau)}}, \frac{\beta+\alpha \int_{a}^{t} \frac{\Delta \tau}{p(\tau)}}{\beta+\alpha \int_{a}^{\sigma(b)} \frac{\Delta \tau}{p(\tau)}}\right\} \in[0,1] .
$$

For the remainder of this paper set

$$
D:=d\left[d-\alpha \sum_{i=1}^{n} \psi_{i}+\gamma \sum_{i=1}^{n} \phi_{i}\right] .
$$

Lemma 2.3 ([6, Lemma 5.1]). Assume (1.4) and (1.5). Let $u \in C_{l d}[a, b]_{\mathbb{T}}$. If $D \neq 0$, then the nonhomogeneous dynamic equation (2.1) with boundary conditions (1.3) has a unique solution $\zeta$ for which the formula

$$
\zeta(t)=\int_{a}^{b} G(t, s) u(s) \nabla s+A(u)\left(\beta+\alpha \int_{a}^{t} \frac{\Delta \tau}{p(\tau)}\right)+B(u)\left(\delta+\gamma \int_{t}^{\sigma(b)} \frac{\Delta \tau}{p(\tau)}\right)
$$

for $t \in[a, \sigma(b)]_{\mathbb{T}}$ holds, where the function $G(t, s)$ is the Green function (2.3), and the functionals $A$ and $B$ are defined by

$$
\begin{aligned}
& A(u):=\frac{1}{D}\left|\begin{array}{cc}
\sum_{i=1}^{n} \psi_{i} \int_{a}^{b} p\left(t_{i}\right) G^{\Delta_{t}}\left(t_{i}, s\right) u(s) \nabla s & \gamma \sum_{i=1}^{n} \psi_{i} \\
\sum_{i=1}^{n} \phi_{i} \int_{a}^{b} p\left(t_{i}\right) G^{\Delta_{t}}\left(t_{i}, s\right) u(s) \nabla s & d+\gamma \sum_{i=1}^{n} \phi_{i}
\end{array}\right|, \\
& B(u):=\frac{1}{D}\left|\begin{array}{cc}
\sum_{i=1}^{n} \psi_{i} \int_{a}^{b} p\left(t_{i}\right) G^{\Delta_{t}}\left(t_{i}, s\right) u(s) \nabla s & -d+\alpha \sum_{i=1}^{n} \psi_{i} \\
\sum_{i=1}^{n} \phi_{i} \int_{a}^{b} p\left(t_{i}\right) G^{\Delta_{t}}\left(t_{i}, s\right) u(s) \nabla s & \alpha \sum_{i=1}^{n} \phi_{i}
\end{array}\right| .
\end{aligned}
$$

Lemma 2.4 ([6, Lemma 5.2]). Assume (1.4) and (1.5). Let $u \in C_{l d}[a, b]_{\mathbb{T}}$ with $u \geq 0$. If

$$
D \neq 0, \quad A(u)>0, \text { and } B(u)>0,
$$

the unique solution $\zeta$ as in (2.5) of the time scale boundary value problem (2.1), (1.3) satisfies

$$
g(t)\|\zeta\| \leq \zeta(t) \leq\|\zeta\|, \quad t \in[a, \sigma(b)]_{\mathbb{T}},
$$

where $g$ is given in (2.4).

\section{Main results}

Theorem 3.1. Assume $\left(H_{1}\right)-\left(H_{3}\right)$ hold. Then, the BVP (1.1) and (1.2) has a unique positive solution $\tilde{y}(t)$ in $\Omega$, where

$$
\Omega=\left\{y \in \mathbb{B} \mid y(t)>0, t \in[a, \sigma(b)]_{\mathbb{T}}\right\} .
$$


Moreover, for any $y_{0} \in \Omega$, constructing successively the sequence

$$
y_{n+1}(t)=\int_{a}^{b} G(t, s) h(s) f\left(y_{n}(s)\right) \nabla s, \quad n=0,1,2, \ldots,
$$

we have $y_{n}(t)$ converges uniformly to $\tilde{y}(t)$ in $[a, \sigma(b)]_{\mathbb{T}}$.

Proof. First, we show that the BVP (1.1) and (1.2) has a solution.

It is easy to see that the BVP (1.1) and (1.2) has a solution $y=y(t)$ if and only if $y$ is a fixed point of the operator equation

$$
S y(t)=\int_{a}^{b} G(t, s) h(s) f(y(s)) \nabla s, \quad t \in[a, \sigma(b)]_{\mathbb{T}} .
$$

In view of $\left(H_{3}\right)$ and (3.1), $S y$ is nonincreasing in $y$. Moreover, for any $\tau \in$ $(a, b)_{\mathbb{T}}$, we have

$$
\begin{aligned}
S\left(\frac{1}{\varphi(\tau)} y\right)(t) & =\int_{a}^{b} G(t, s) h(s) f\left(\frac{1}{\varphi(\tau)} y(s)\right) \nabla s \\
& \geq \omega(\tau) \int_{a}^{b} G(t, s) h(s) f(y(s)) \nabla s=\omega(\tau) S y(t)
\end{aligned}
$$

for $t \in[a, \sigma(b)]_{\mathbb{T}}$ and $y \in \mathbb{B}$ with $y \geq 0$.

Let $L=\int_{a}^{b} G(s, s) h(s) \nabla s$, the condition $\left(H_{2}\right)$ implies $L>0$. Since $f(y)>0$ for $y>0$, by Lemma 2.2 , we have

$$
\begin{aligned}
S(L) & =\int_{a}^{b} G(t, s) h(s) f(L) \nabla s \\
& =f(L) \int_{a}^{b} G(t, s) h(s) \nabla s \\
& \geq f(L) g(t) \int_{a}^{b} G(s, s) h(s) \nabla s \\
& =f(L) g(t) L, \quad t \in[a, \sigma(b)]_{\mathbb{T}} .
\end{aligned}
$$

Moreover, we obtain

$$
\begin{aligned}
S(L) & \leq f(L) \int_{a}^{b} G(s, s) h(s) \nabla s \\
& =f(L) L .
\end{aligned}
$$

Thus

$$
f(L) g(t) L \leq S(L) \leq f(L) L, \quad t \in[a, \sigma(b)]_{\mathbb{T}} .
$$

Therefore, we can choose a sufficiently small number $e \in(0,1)$ such that

$$
e L \leq S(L) \leq \frac{L}{e}
$$


It follows from $\left(H_{3}\right)$ that there exists $\tau_{0} \in(a, b)_{\mathbb{T}}$ such that $\varphi\left(\tau_{0}\right)=e$, so

$$
\varphi\left(\tau_{0}\right) L \leq S(L) \leq \frac{L}{\varphi\left(\tau_{0}\right)}
$$

Since $\frac{\omega\left(\tau_{0}\right)}{\varphi\left(\tau_{0}\right)}>1$, we can take a sufficiently large positive integer $k$ such that

$$
\left[\frac{\omega\left(\tau_{0}\right)}{\varphi\left(\tau_{0}\right)}\right]^{k} \geq \frac{1}{\varphi\left(\tau_{0}\right)}
$$

It is clear that

$$
\left[\frac{\varphi\left(\tau_{0}\right)}{\omega\left(\tau_{0}\right)}\right]^{k} \leq \varphi\left(\tau_{0}\right)
$$

We define

$$
u_{0}=\left[\varphi\left(\tau_{0}\right)\right]^{k} L, \quad v_{0}=\frac{L}{\left[\varphi\left(\tau_{0}\right)\right]^{k}} .
$$

Evidently, $u_{0}=\left[\varphi\left(\tau_{0}\right)\right]^{2 k} v_{0}<v_{0}$. Take any $\lambda \in\left(0,\left[\varphi\left(\tau_{0}\right)\right]^{2 k}\right]$, then $\lambda \in(0,1)$ and $u_{0} \geq \lambda v_{0}$.

By the monotonicity of $S$, we have $S v_{0} \leq S u_{0}$. Furthermore, combining $\left(H_{3}\right)$ with $(3.3)$ and $(3.4)$, we have

$$
\begin{aligned}
S v_{0} & =S\left(\frac{1}{\left[\varphi\left(\tau_{0}\right)\right]^{k}} L\right)=S\left(\frac{1}{\varphi\left(\tau_{0}\right)\left[\varphi\left(\tau_{0}\right)\right]^{k-1}} L\right) \\
& \geq \omega\left(\tau_{0}\right) S\left(\frac{1}{\left[\varphi\left(\tau_{0}\right)\right]^{k-1}} L\right) \geq \cdots \\
& \geq\left[\omega\left(\tau_{0}\right)\right]^{k} S(L) \geq\left[\omega\left(\tau_{0}\right)\right]^{k} \varphi\left(\tau_{0}\right) L \geq\left[\varphi\left(\tau_{0}\right)\right]^{k} L=u_{0} .
\end{aligned}
$$

From $\left(H_{3}\right)$, we get

$$
S y=S\left(\frac{1}{\varphi(s)} \varphi(s) y\right) \geq \omega(s) S(\varphi(s) y), \quad \forall s \in(a, b)_{\mathbb{T}}, \quad y \geq 0,
$$

and hence

Thus, we have

$$
S(\varphi(s) y) \leq \frac{1}{\omega(s)} S y, \quad \forall s \in(a, b)_{\mathbb{T}}, \quad y \geq 0 .
$$

$$
\begin{aligned}
S u_{0} & =S\left(\left[\varphi\left(\tau_{0}\right)\right]^{k} L\right)=S\left(\varphi\left(\tau_{0}\right)\left[\varphi\left(\tau_{0}\right)\right]^{k-1} L\right) \leq \frac{1}{\omega\left(\tau_{0}\right)} S\left(\left[\varphi\left(\tau_{0}\right)\right]^{k-1} L\right) \\
& \leq \cdots \leq \frac{1}{\left[\omega\left(\tau_{0}\right)\right]^{k}} S(L) \leq \frac{1}{\left[\omega\left(\tau_{0}\right)\right]^{k}} \frac{L}{\varphi\left(\tau_{0}\right)} .
\end{aligned}
$$

An application of (3.5) yields

$$
S u_{0} \leq \frac{L}{\left[\varphi\left(\tau_{0}\right)\right]^{k}}=v_{0} .
$$

Constructing successively the sequences

$$
u_{n}=S v_{n-1}, \quad v_{n}=S u_{n-1}, \quad n=1,2, \ldots
$$


By the monotonicity of $S$, we have $u_{1}=S v_{0} \leq S u_{0}=v_{1}$. By induction, we obtain $u_{n} \leq v_{n}, \quad n=1,2, \ldots$. It follows from (3.6), (3.7) and the monotonicity of $S$ that

$$
u_{0} \leq u_{1} \leq \cdots \leq u_{n} \leq \cdots \leq v_{n} \leq \cdots \leq v_{1} \leq v_{0} .
$$

Note that $u_{0} \geq \lambda v_{0}$, so we can get $u_{n} \geq u_{0} \geq \lambda v_{0} \geq \lambda v_{n}, \quad n=1,2, \ldots$. Let

$$
\lambda_{n}=\sup \left\{\lambda>0 \mid u_{n} \geq \lambda v_{n}\right\}, \quad n=1,2, \ldots
$$

Thus, we have

$$
u_{n} \geq \lambda_{n} v_{n}, n=1,2, \ldots
$$

and then

$$
u_{n+1} \geq u_{n} \geq \lambda_{n} v_{n} \geq \lambda_{n} v_{n+1}, \quad n=1,2, \ldots
$$

Therefore, $\lambda_{n+1} \geq \lambda_{n}$, i.e., $\left\{\lambda_{n}\right\}$ is increasing with $\left\{\lambda_{n}\right\} \subset(0,1]$. Set $\lambda^{*}=$ $\lim _{n \rightarrow \infty} \lambda_{n}$. We can prove that $\lambda^{*}=1$. In fact, if $0<\lambda^{*}<1$, by $\left(H_{3}\right)$, there exists $\bar{\tau}_{0} \in(a, b)_{\mathbb{T}}$ such that $\varphi\left(\bar{\tau}_{0}\right)=\lambda^{*}$. Consider the following two cases:

(i) There exists an integer $N$ such that $\lambda_{N}=\lambda^{*}$. In this case, we have $\lambda_{n}=\lambda^{*}$ for all $n \geq N$ holds. Hence, for $n \geq N$, it follows from (3.2) and the monotonicity of $S$ that

$$
u_{n+1}=S v_{n} \geq S\left(\frac{1}{\lambda^{*}} u_{n}\right)=S\left(\frac{1}{\varphi\left(\bar{\tau}_{0}\right)} u_{n}\right) \geq \omega\left(\bar{\tau}_{0}\right) S u_{n}=\omega\left(\bar{\tau}_{0}\right) v_{n+1} .
$$

By the definition of $\lambda_{n}$, we have

$$
\lambda_{n+1}=\lambda^{*} \geq \omega\left(\bar{\tau}_{0}\right)>\varphi\left(\bar{\tau}_{0}\right)=\lambda^{*} .
$$

This is a contradiction.

(ii) For all integer $n, \lambda_{n}<\lambda^{*}$. In this case, we have $0<\frac{\lambda_{n}}{\lambda^{*}}<1$. In virtue of $\left(H_{3}\right)$, there exists $\mu_{n} \in(a, b)_{\mathbb{T}}$ such that $\varphi\left(\mu_{n}\right)=\frac{\lambda_{n}}{\lambda^{*}}$. Hence, combining (3.2) with the monotonicity of $S$, we have

$$
\begin{aligned}
u_{n+1} & =S v_{n} \geq S\left(\frac{1}{\lambda_{n}} u_{n}\right)=S\left(\frac{1}{\frac{\lambda_{n}}{\lambda^{*}}} \cdot \frac{1}{\lambda^{*}} u_{n}\right)=S\left(\frac{1}{\varphi\left(\mu_{n}\right)} \cdot \frac{1}{\varphi\left(\bar{\tau}_{0}\right)} u_{n}\right) \\
& \geq \omega\left(\mu_{n}\right) S\left(\frac{1}{\varphi\left(\bar{\tau}_{0}\right)} u_{n}\right) \geq \omega\left(\mu_{n}\right) \omega\left(\bar{\tau}_{0}\right) S u_{n} \\
& =\omega\left(\mu_{n}\right) \omega\left(\bar{\tau}_{0}\right) v_{n+1} .
\end{aligned}
$$

By the definition of $\lambda_{n}$, we have

$$
\lambda_{n+1} \geq \omega\left(\mu_{n}\right) \omega\left(\bar{\tau}_{0}\right)>\varphi\left(\mu_{n}\right) \omega\left(\bar{\tau}_{0}\right)=\frac{\lambda_{n}}{\lambda^{*}} \omega\left(\bar{\tau}_{0}\right) .
$$

Let $n \longrightarrow \infty$, we have $\lambda^{*} \geq \frac{\lambda^{*}}{\lambda^{*}} \omega\left(\bar{\tau}_{0}\right)>\frac{\lambda^{*}}{\lambda^{*}} \varphi\left(\bar{\tau}_{0}\right)=\varphi\left(\bar{\tau}_{0}\right)=\lambda^{*}$, and this is also a contradiction. Hence, $\lim _{n \rightarrow \infty} \lambda_{n}=1$.

Thus, combining (3.8) with (3.9), we have

$$
0 \leq u_{n+l}(t)-u_{n}(t) \leq v_{n}(t)-\lambda_{n} v_{n}(t)=\left(1-\lambda_{n}\right) v_{n}(t) \leq\left(1-\lambda_{n}\right) v_{0}(t)
$$


for $t \in[a, \sigma(b)]_{\mathbb{T}}$, where $l$ is a nonnegative integer. Thus,

$$
\left\|u_{n+l}-u_{n}\right\| \leq\left\|v_{n}-u_{n}\right\| \leq\left(1-\lambda_{n}\right) v_{0} .
$$

Therefore, there exists a positive function $\tilde{y} \in \mathbb{B}$ such that

$$
\lim _{n \rightarrow \infty} u_{n}(t)=\lim _{n \rightarrow \infty} v_{n}(t)=\tilde{y}(t) \text { for } t \in[a, \sigma(b)]_{\mathbb{T}} .
$$

By the monotonicity of $S$ and (3.8), we have

$$
u_{n+1}(t)=S v_{n}(t) \leq S \tilde{y}(t) \leq S u_{n}(t)=v_{n+1}(t) .
$$

Let $n \longrightarrow \infty$ and we get $S \tilde{y}(t)=\tilde{y}(t)$. That is, $\tilde{y}(t)$ is a positive solution of the BVP (1.1) and (1.2).

Next, we show the uniqueness of solutions of the BVP (1.1) and (1.2). Assume, to the contrary, that there exists a positive solution $\bar{y}(t)$ of the BVP (1.1) and (1.2) such that $\bar{y}(t) \neq \tilde{y}(t), t \in[a, \sigma(b)]_{\mathbb{T}}$. We can know that there exists $0<\eta \leq 1$ such that $\eta \tilde{y}(t) \leq \bar{y}(t) \leq \frac{1}{\eta} \tilde{y}(t)$ for $t \in[a, \sigma(b)]_{\mathbb{T}}$. Let

$$
\eta_{0}=\sup \left\{0<\eta \leq 1 \mid \eta \tilde{y}(t) \leq \bar{y}(t) \leq \frac{1}{\eta} \tilde{y}(t), \quad t \in[a, \sigma(b)]_{\mathbb{T}}\right\} .
$$

Then $0<\eta_{0} \leq 1$ and $\eta_{0} \tilde{y}(t) \leq \bar{y}(t) \leq \frac{1}{\eta_{0}} \tilde{y}(t)$ for $t \in[a, \sigma(b)]_{\mathbb{T}}$.

We now show that $\eta_{0}=1$. In fact, if $0<\eta_{0}<1$, then, in view of $\left(H_{3}\right)$, there exists $\tau_{1} \in(a, b)_{\mathbb{T}}$ such that $\varphi\left(\tau_{1}\right)=\eta_{0}$. Furthermore, we have

$$
\bar{y}=S \bar{y} \geq S\left(\frac{1}{\eta_{0}} \tilde{y}\right)=S\left(\frac{1}{\varphi\left(\tau_{1}\right)} \tilde{y}\right) \geq \omega\left(\tau_{1}\right) S \tilde{y}=\omega\left(\tau_{1}\right) \tilde{y}
$$

and

$$
\bar{y}=S \bar{y} \leq S\left(\eta_{0} \tilde{y}\right) \leq \frac{1}{\omega\left(\tau_{1}\right)} S\left(\frac{\eta_{0} \tilde{y}}{\varphi\left(\tau_{1}\right)}\right)=\frac{1}{\omega\left(\tau_{1}\right)} S \tilde{y}=\frac{1}{\omega\left(\tau_{1}\right)} \tilde{y} .
$$

Since $\omega\left(\tau_{1}\right)>\varphi\left(\tau_{1}\right)=\eta_{0}$, this contradicts the definition of $\eta_{0}$. Hence $\eta_{0}=1$. Therefore, the BVP (1.1) and (1.2) has a unique solution.

Finally, we show that "moreover" part of the theorem. For any initial $y_{0} \in \Omega$, we can choose a sufficiently small number $\bar{e} \in(0,1)$ such that

$$
\bar{e} L \leq y_{0} \leq \frac{1}{\bar{e}} L .
$$

It follows from $\left(H_{3}\right)$ that there exists $\tau_{2} \in(a, b)_{\mathbb{T}}$ such that $\varphi\left(\tau_{2}\right)=\bar{e}$; and hence

$$
\varphi\left(\tau_{2}\right) L \leq y_{0} \leq \frac{L}{\varphi\left(\tau_{2}\right)} .
$$

We can choose a sufficiently large positive integer $k$ such that

$$
\left[\frac{\omega\left(\tau_{2}\right)}{\varphi\left(\tau_{2}\right)}\right]^{k} \geq \frac{1}{\varphi\left(\tau_{2}\right)}
$$

Define

$$
\hat{u}_{0}=\left[\varphi\left(\tau_{2}\right)\right]^{k} L, \quad \hat{v}_{0}=\frac{L}{\left[\varphi\left(\tau_{2}\right)\right]^{k}} .
$$


Obviously, $\hat{u}_{0}<y_{0}<\hat{v}_{0}$. Let

$$
\begin{gathered}
\hat{u}_{n}=S \hat{v}_{n-1}, \quad \hat{v}_{n}=S \hat{u}_{n-1}, \\
y_{n}(t)=S y_{n-1}(t)=\int_{a}^{b} G(t, s) h(s) f\left(y_{n-1}(t)\right) \nabla s,
\end{gathered}
$$

for $t \in[a, \sigma(b)]_{\mathbb{T}}$, and $n=1,2, \ldots$ By induction, we get $\hat{u}_{n} \leq y_{n} \leq \hat{v}_{n}$, $n=1,2, \ldots$.

Similarly to the above proof, it follows that there exists $y^{*} \in \Omega$ such that

$$
\lim _{n \rightarrow \infty} \hat{u}_{n}=\lim _{n \rightarrow \infty} \hat{v}_{n}=y^{*}, \quad S y^{*}=y^{*} .
$$

By the uniqueness of fixed points of $S$ in $\Omega$, we get $y^{*}=\tilde{y}$. Therefore, we have

$$
\lim _{n \rightarrow \infty} \sup _{t \in[a, \sigma(b)]_{\mathbb{T}}}\left|y_{n}(t)-\tilde{y}(t)\right|=0 .
$$

This completes the proof of the theorem.

Remark 3.1. In Theorem 3.1, we give a way to find the solutions which will be useful from an application viewpoint. However, whether this method can be extended to the semipositone problem is still an open problem.

Similarly to the proof of Theorem 3.1, we can obtain the following result by means of Lemma 2.3 and Lemma 2.4.

Theorem 3.2. Let $d-\alpha \sum_{i=1}^{n} \psi_{i}>0, \psi_{j} d+\gamma \psi_{j} \sum_{i=1}^{n} \phi_{i}-\gamma \phi_{j} \sum_{i=1}^{n} \psi_{i}>$ $0(j=1,2, \ldots, n)$. Assume $\left(H_{1}\right)-\left(H_{3}\right)$ hold. Then, the $B V P(1.1)$ and $(1.3)$ has a unique positive solution $y^{*}(t)$ in $\Omega$. Moreover, for any $y_{0} \in \Omega$, constructing successively the sequence

$$
\begin{aligned}
y_{n+1}(t)= & \int_{a}^{b} G(t, s) h(s) f\left(y_{n}(s)\right) \nabla s+A\left(h(s) f\left(y_{n}(s)\right)\right)\left(\beta+\alpha \int_{a}^{t} \frac{\Delta \tau}{p(\tau)}\right) \\
& +B\left(h(s) f\left(y_{n}(s)\right)\right)\left(\delta+\gamma \int_{t}^{\sigma(b)} \frac{\Delta \tau}{p(\tau)}\right)
\end{aligned}
$$

for $t \in[a, \sigma(b)]_{\mathbb{T}}, n=0,1,2, \ldots$, we have $y_{n}(t)$ converges uniformly to $y^{*}(t)$ in $[a, \sigma(b)]_{\mathbb{T}}$.

In Theorem 3.1, let $f=f_{1}+f_{2}$, under appropriate conditions, we have the following theorem which generalizes and extends Theorem 3.1.

Theorem 3.3. Assume $\left(H_{2}\right)$ holds, and the following conditions are satisfied $\left(C_{1}\right) f_{i}:[0,+\infty) \longrightarrow[0,+\infty)$ are continuous and $f_{i}(y)>0$ for $y>0(i=1,2)$; $\left(C_{2}\right) f_{1}:[0,+\infty) \longrightarrow[0,+\infty)$ is nondecreasing, $f_{2}:[0,+\infty) \longrightarrow[0,+\infty)$ is nonincreasing, and there exist two positive-value functions $\varphi(\tau), \omega(\tau)$ on 
interval $(a, b)_{\mathbb{T}}$ such that $\varphi:(a, b)_{\mathbb{T}} \longrightarrow(0,1)$ is a surjection and $\omega(\tau)>$ $\varphi(\tau), \forall \tau \in(a, b)_{\mathbb{T}}$ which satisfy

$$
f_{1}(\varphi(s) y) \geq \omega(s) f_{1}(y), \quad f_{2}\left(\frac{1}{\varphi(s)} y\right) \geq \omega(s) f_{2}(y)
$$

$\forall s \in(a, b)_{\mathbb{T}}, y \in[0,+\infty)$. Then, the BVP (1.1) and (1.2) has a unique positive solution $y^{*}(t)$ in $\Omega$. Moreover, for any $x_{0}, y_{0} \in \Omega$, constructing successively the sequences

$$
\begin{aligned}
& x_{n+1}(t)=\int_{a}^{b} G(t, s) h(s)\left[f_{1}\left(x_{n}(s)\right)+f_{2}\left(y_{n}(s)\right)\right] \nabla s, \quad t \in[a, \sigma(b)]_{\mathbb{T}}, \\
& y_{n+1}(t)=\int_{a}^{b} G(t, s) h(s)\left[f_{1}\left(y_{n}(s)\right)+f_{2}\left(x_{n}(s)\right)\right] \nabla s, \quad t \in[a, \sigma(b)]_{\mathbb{T}},
\end{aligned}
$$

$n=0,1,2, \ldots$, we have

$$
\lim _{n \rightarrow \infty} \sup _{t \in[a, \sigma(b)]_{\mathbb{T}}}\left|x_{n}(t)-y^{*}(t)\right|=0, \quad \lim _{n \rightarrow \infty} \sup _{t \in[a, \sigma(b)]_{\mathbb{T}}}\left|y_{n}(t)-y^{*}(t)\right|=0 .
$$

In the following, we will apply Theorem 3.3 to the BVP (1.13) and (1.14), our conclusion is the following theorem.

Theorem 3.4. Assume the following conditions are satisfied

$\left(D_{1}\right) f=f_{1}+f_{2}$, where $f_{i}:[a, b]_{\mathbb{T}} \times[0,+\infty) \longrightarrow[0,+\infty)$ are continuous $(i=1,2)$;

$\left(D_{2}\right)$ for fixed $t \in[a, b]_{\mathbb{T}}, f_{1}(t, u)$ is increasing on $u$, and $f_{2}(t, u)$ is decreasing on $u$;

$\left(D_{3}\right)$ there exist two positive-value functions $\varphi(\tau), \omega(\tau)$ on interval $(a, b)_{\mathbb{T}}$ such that $\varphi:(a, b)_{\mathbb{T}} \longrightarrow(0,1)$ is a surjection and $\omega(\tau)>\varphi(\tau), \forall \tau \in(a, b)_{\mathbb{T}}$ which satisfy

$$
f_{1}(t, \varphi(s) u) \geq \omega(s) f_{1}(t, u), f_{2}\left(t, \frac{1}{\varphi(s)} u\right) \geq \omega(s) f_{2}(t, u)
$$

for $s \in(a, b)_{\mathbb{T}}$ and $(t, u) \in[a, b]_{\mathbb{T}} \times[0,+\infty)$;

$\left(D_{4}\right) \int_{a}^{\sigma(b)} \frac{\delta \tilde{G}(\eta, s)}{\sigma^{2}(b)-a-\delta(\eta-a)}\left[f_{1}(s, s-a)+f_{2}(s, s-a)\right] \Delta s>0$, where $\tilde{G}(t, s)$ is defined in (3.10). Then, the BVP (1.13) and (1.14) has a unique positive solution $x^{*}(t)$ in $\bar{\Omega}$, where

$$
\begin{gathered}
\bar{\Omega}=\left\{x(t) \in C\left[a, \sigma^{2}(b)\right]_{\mathbb{T}} \mid \exists M \geq m>0 \text { such that } m(t-a) \leq x(t)\right. \\
\left.\leq M(t-a), \quad t \in\left[a, \sigma^{2}(b)\right]_{\mathbb{T}}\right\} .
\end{gathered}
$$

Furthermore, for any $x_{0}, y_{0} \in \bar{\Omega}$, constructing successively the sequences

$$
x_{n+1}(t)=\int_{a}^{\sigma(b)} K(t, s)\left[f_{1}\left(s, x_{n}(s)\right)+f_{2}\left(s, y_{n}(s)\right)\right] \Delta s,
$$




$$
y_{n+1}(t)=\int_{a}^{\sigma(b)} K(t, s)\left[f_{1}\left(s, y_{n}(s)\right)+f_{2}\left(s, x_{n}(s)\right)\right] \Delta s
$$

for $t \in\left[a, \sigma^{2}(b)\right]_{\mathbb{T}}$, and $n=0,1,2, \ldots$, where

$$
K(t, s)=\tilde{G}(t, s)+\frac{\delta \tilde{G}(\eta, s)}{\sigma^{2}(b)-a-\delta(\eta-a)}(t-a)
$$

with

$$
\tilde{G}(t, s)=\frac{1}{\sigma^{2}(b)-a} \begin{cases}(t-a)\left(\sigma^{2}(b)-\sigma(s)\right), & t \leq s, \\ (\sigma(s)-a)\left(\sigma^{2}(b)-t\right), & t \geq \sigma(s),\end{cases}
$$

we have

$$
\lim _{n \rightarrow \infty} \sup _{t \in\left[a, \sigma^{2}(b)\right]_{\mathbb{T}}}\left|x_{n}(t)-x^{*}(t)\right|=0, \quad \lim _{n \rightarrow \infty} \sup _{t \in\left[a, \sigma^{2}(b)\right]_{\mathbb{T}}}\left|y_{n}(t)-x^{*}(t)\right|=0 .
$$

Proof of Theorem 3.4. Let

$$
\begin{gathered}
E=\left\{x \mid x:\left[a, \sigma^{2}(b)\right]_{\mathbb{T}} \longrightarrow \mathbb{R} \text { is continuous }\right\}, \\
P=\left\{x \in E \mid x(t) \geq 0 \text { for } t \in\left[a, \sigma^{2}(b)\right]_{\mathbb{T}}\right\} .
\end{gathered}
$$

Define an operator $F: P \times P \longrightarrow E$ by

$$
F\left(x_{1}, x_{2}\right)(t)=\int_{a}^{\sigma(b)} K(t, s)\left[f_{1}\left(s, x_{1}(s)\right)+f_{2}\left(s, x_{2}(s)\right)\right] \Delta s, \quad t \in\left[a, \sigma^{2}(b)\right]_{\mathbb{T}} .
$$

According to Lemma 2.1 in [22], we can know that $x$ is a solution of problem (1.13) and (1.14) if and only if $x=F(x, x)$. By condition $\left(D_{1}\right)$, we can obtain that $F: P \times P \longrightarrow P$.

Similarly to the proof of Theorem 3.1 in [21], it suffices to verify that $F(t-$ $a, t-a) \in \bar{\Omega}$.

In fact, for any $t \in\left[a, \sigma^{2}(b)\right]_{\mathbb{T}}$, it follows from Lemma 2.2 in [22] that

$$
\begin{aligned}
& F(t-a, t-a) \\
= & \int_{a}^{\sigma(b)} K(t, s)\left[f_{1}(s, s-a)+f_{2}(s, s-a)\right] \Delta s \\
\geq & \int_{a}^{\sigma(b)} \frac{\delta \tilde{G}(\eta, s)(t-a)}{\sigma^{2}(b)-a-\delta(\eta-a)}\left[f_{1}(s, s-a)+f_{2}(s, s-a)\right] \Delta s \\
= & \int_{a}^{\sigma(b)} \frac{\delta \tilde{G}(\eta, s)}{\sigma^{2}(b)-a-\delta(\eta-a)}\left[f_{1}(s, s-a)+f_{2}(s, s-a)\right] \Delta s(t-a),
\end{aligned}
$$

and

(3.12) $F(t-a, t-a)$

$$
\leq \int_{a}^{\sigma(b)}\left[1+\frac{\delta \tilde{G}(\eta, s)}{\sigma^{2}(b)-a-\delta(\eta-a)}\right]\left[f_{1}(s, s-a)+f_{2}(s, s-a)\right] \Delta s(t-a) .
$$

Combining condition $\left(D_{4}\right)$ with (3.11) and (3.12), we have $F(t-a, t-a) \in \bar{\Omega}$.

The rest proof is similar to that of Theorem 3.1 in [21], we omit it here. 
Remark 3.2. In Theorem 3.4, we impose the more general conditions on the function $f$ than the conditions in Theorem 1.1. Moreover, the uniqueness of positive solutions of the BVP (1.13) and (1.14) is obtained, and the iterative sequences which converge uniformly to approximation solutions are also given.

In the end, we give an example to illustrate our results.

Example 3.1. Let $\mathbb{T}=\left\{2^{k}\right\}_{k \in \mathbb{Z}} \cup\{0\}$, where $\mathbb{Z}$ denotes the set of all integers. Considering the following BVP

$$
\begin{gathered}
y^{\Delta \nabla}(t)+\sum_{i=1}^{n} \beta_{i} y^{-\alpha_{i}}=0, \quad t \in(0,1]_{\mathbb{T}}, \\
y(0)-y^{\Delta}(0)=0, \quad y^{\sigma}(1)+y^{\Delta}(1)=0,
\end{gathered}
$$

where $n$ is a positive integer, $\alpha_{i} \in[0,1)$ and $\beta_{i}>0$ for $i=1,2, \ldots, n$. We claim that, the BVP (3.13) and (3.14) has a unique positive solution $\tilde{y}(t)$. Furthermore, for any $y_{0} \in D$, constructing successively the sequence

$$
y_{n+1}(t)=\int_{0}^{1} G(t, s) h(s) \sum_{i=1}^{n} \beta_{i}\left(y_{n}(s)\right)^{-\alpha_{i}} \nabla s
$$

for $t \in[0,1]_{\mathbb{T}}$ and $n=0,1,2, \ldots$, we have

$$
\lim _{n \rightarrow \infty} \sup _{t \in[0,1]_{\mathbb{T}}}\left|y_{n}(t)-\tilde{y}(t)\right|=0 \text {. }
$$

In fact, by direct computation, we get

$$
\begin{aligned}
\int_{0}^{1} G(s, s) \nabla s & =4+\sum_{n=0}^{\infty} 2^{-1-2 n}-\sum_{n=0}^{\infty} 2^{-3-3 n}-\sum_{n=0}^{\infty} 2^{-2 n}+\sum_{n=0}^{\infty} 2^{-1-3 n} \\
& =\frac{79}{21}>0
\end{aligned}
$$

Note that for $y \in(0,+\infty), f(y)=\sum_{i=1}^{n} \beta_{i} y^{-\alpha_{i}}>0$ and is nonincreasing. We set $\varphi(\tau)=\tau, \omega(\tau)=\tau^{\theta}\left(\theta \in\left(\sup _{i} \alpha_{i}, 1\right)\right)$. Moreover, for $\theta \in\left(\sup _{i} \alpha_{i}, 1\right)$, it is easy to see that $\varphi:(0,1)_{\mathbb{T}} \longrightarrow(0,1)$ is a surjection and $\omega(\tau)>\varphi(\tau)$ for $\tau \in(0,1)_{\mathbb{T}}$. For $y \geq 0$, we have

$$
f\left(\frac{1}{s} y\right)=\sum_{i=1}^{n} \beta_{i}\left(\frac{1}{s} y\right)^{-\alpha_{i}} \geq s^{\theta} \sum_{i=1}^{n} \beta_{i} y^{-\alpha_{i}}=s^{\theta} f(y), \quad s \in(0,1)_{\mathbb{T}}
$$

i.e., the condition $\left(H_{3}\right)$ in Theorem 3.1 holds. The conclusion then follows from Theorem 3.1.

\section{References}

[1] R. P. Agarwal and D. O'Regan, Nonlinear boundary value problems on time scales, Nonlinear Anal. 44 (2001), no. 4, 527-535. 
[2] R. P. Agarwal, V. Otero-Espinar, K. Perera, and D. R. Vivero, Multiple positive solutions of singular Dirichlet problems on time scales via variational methods, Nonlinear Anal. 67 (2007), no. 2, 368-381.

[3] D. R. Anderson, Solutions to second-order three-point problems on time scales, J. Difference Equ. Appl. 8 (2002), no. 8, 673-688.

[4] _ Existence of solutions for nonlinear multi-point problems on time scales, Dynam. Syst. Appl. 15 (2006), no. 1, 21-33.

[5] D. R. Anderson and R. Y. Ma, Second-order n-point eigenvalue problems on time scales, Adv. Difference Equ. 2006 (2006), Art. ID 59572, 17 pp.

[6] D. R. Anderson and P. J. Y. Wong, Positive solutions for second-order semipositone problems on time scales, Comput. Math. Appl. 58 (2009), no. 2, 281-291.

[7] D. R. Anderson and C. B. Zhai, Positive solutions to semi-positone second-order threepoint problems on time scales, Appl. Math. Comput. 215 (2010), no. 10, 3713-3720.

[8] F. M. Atici, D. C. Biles, and A. Lebedinsky, An application of time scales to economics, Math. Comput. Modelling 43 (2006), no. 7-8, 718-726.

[9] F. M. Atici and G. Sh. Guseinov, On Green's functions and positive solutions for boundary value problems on time scales, J. Comput. Appl. Math. 141 (2002), no. 1-2, 75-99.

[10] M. Bohner and A. Peterson, Dynamic Equations on Time Scales: An Introduction with Applications, Birkhäuser, Boston, 2001.

[11] _ Advances in Dynamic Equations on Time Scales, Birkhäuser, Boston, 2003.

[12] J. J. Dacunha, J. M. Davis, and P. K. Singh, Existence results for singular three point boundary value problems on time scales, J. Math. Anal. Appl. 295 (2004), no. 2, 378391.

[13] N. A. Hamal and F. Yoruk, Positive solutions of nonlinear m-point boundary value problems on time scales, J. Comput. Appl. Math. 231 (2009), no. 1, 92-105.

[14] S. Hilger, Analysis on measure chains-a unified approach to continuous and discrete calculus, Results Math. 18 (1990), no. 1-2, 18-56.

[15] V. Jamieson and V. Spedding, Taming nature's numbers, New Scientist: the global science and technology weekly 2404 (2003), 28-31.

[16] T. Jankowski, On dynamic equations with deviating arguments, Appl. Math. Comput. 208 (2009), no. 2, 423-426.

[17] E. R. Kaufmann, Positive solutions of a three-point boundary value problem on a time scale, Electron. J. Differential Equations 2003 (2003), no. 82, 1-11.

[18] Z. Liang, Existence and uniqueness of fixed points for mixed monotone operators, Journal of Dezhou University 24 (2008), no. 4, 1-6 (in Chinese).

[19] H. Luo, Positive solutions to singular multi-point dynamic eigenvalue problems with mixed derivatives, Nonlinear Anal. 70 (2009), no. 4, 1679-1691.

[20] Y. Sang, Successive iteration and positive solutions for nonlinear m-point boundary value problems on time scales, Discrete Dyn. Nat. Soc. 2009 (2009), Article ID 618413, 13 pages.

[21] Y. Sang, Z. Wei, and W. Dong, Existence and uniqueness of positive solutions for discrete fourth-order Lidstone problem with a parameter, Adv. Difference Equ. 2010 (2010), Article ID 971540, 18 pages.

[22] J. Sun, Existence of positive solution to second-order three-point BVPs on time scales, Bound. Value Probl. 2009 (2009), Article ID 685040, 6 pages.

[23] C. C. Tisdell and A. Zaidi, Basic qualitative and quantitative results for solutions to nonlinear, dynamic equations on time scales with an application to economic modelling, Nonlinear Anal. 68 (2008), no. 11, 3504-3524.

[24] P. Wang, H. Wu, and Y. Wu, Higher even-order convergence and coupled solutions for second-order boundary value problems on time scales, Comput. Math. Appl. 55 (2008), no. 8, 1693-1705. 
[25] C. Zhai and X. Cao, Fixed point theorems for $\tau-\varphi$-concave operators and applications, Comput. Math. Appl. 59 (2010), no. 1, 532-538.

[26] X. Zhao and W. Ge, Multiple positive solutions for time scale boundary value problems on infinite intervals, Acta. Appl. Math. 106 (2009), no. 2, 265-273.

YANBIN SANG

Department of Mathematics

NORTh UNIVERSiTy OF CHINA

Taiyuan, Shanxi, 030051, P. R. China

E-mail address: syb6662004@yahoo.com.cn

ZHONGLI WEI

SCHOOL OF Mathematics

SHANDONG UNIVERSITY

Jinan, Shandong, 250100, P. R. China

E-mail address: jnwzl@yahoo.com.cn

Wei Dong

Hebei University of EngineERing

Handan, Hebei, 056021, P. R. China

E-mail address: wdongau@yahoo.com.cn 